\title{
The Genetic Location of Three Mutations Impairing Penicillin Production in Aspergillus nidulans
}

\author{
By J. F. MAKINS,${ }^{1} \dagger$ G. HOLT ${ }^{1}$ AND K. D. MACDONALD ${ }^{2 *}$ \\ ${ }^{1}$ School of Engineering and Science, Polytechnic of Central London, 115 New Cavendish Street, \\ London W1M 8JS, U.K., \\ ${ }^{2}$ Chemical Defence Establishment, Porton Down, Salisbury, Wiltshire SP4 OJQ, U.K.
}

(Received 23 December 1982; revised 25 April 1983)

Three mutations impairing penicillin production in Aspergillus nidulans, npeB, npeC and npeD, have been located on linkage groups III, IV and II, respectively, and positioned relative to other loci on these chromosomes.

\section{INTRODUCTION}

Both Aspergillus nidulans and Penicillium chrysogenum elaborate penicillin and have been used in genetic and biochemical studies of the biosynthesis and over-production of this antibiotic (Macdonald \& Holt, 1976). Although only producing small amounts, $A$. nidulans has several advantages over $P$. chrysogenum as an organism with which to study the genetics of penicillin production. Foremost are the wealth of genetic information available and the existence of wellcharacterized multiple mutant strains to analyse genetic events in this organism (Clutterbuck, 1974).

Following treatment with a variety of different mutagens, strains of $A$. nidulans with altered abilities to elaborate penicillin have been isolated (Edwards et al., 1974; Holt et al., 1976; Ditchburn et al., 1976). Three mutations which raised penicillin yield, pen $A, B$ and $C$, have been assigned to positions on linkage groups VIII, III and IV, respectively (Ditchburn et al., 1976). Mutants impaired in penicillin production (Npe) have also been isolated and allotted to one of four complementation groups, $A, B, C$ and $D$; over two thirds were members of complementation group $A$ (Edwards et al., 1974). Additionally, the lack of penicillin production in certain wild-type isolates of $\boldsymbol{A}$. nidulans was shown to be the result of changes at this same npeA locus (Cole et al., 1976). The npeA gene was located on linkage group VI (Holt et al., 1976) and subsequent investigations by Makins et al. (1981) and Makins \& Holt (1982) suggested it controlled the formation of the tripeptide $\alpha$-aminoadipylcysteinylvaline, a precursor of penicillin, and was probably analogous to npe $Y$ in $P$. chrysogenum (Normansell et al., 1980). While mutants with lesions at the npe $A$ locus have been the subject of considerable attention, relatively little is known about mutations in complementation groups $B, C$ and $D$. This paper reports studies on strains with mutations in these latter three complementation groups.

\section{METHODS}

Strains. Details are given in Table 1. The gene symbols used are those of Clutterbuck (1974). The criterion for classifying a mutant as Npe was reduction of penicillin yield to $10 \%$ or less that of its parent without affecting nutritional requirements, growth rate or gross morphology.

Media. Minimal media (MM), complete media (CM) and sporulation media (SPM) were based on those of Pontecorvo et al. (1953). The penicillin production media (PPM) was described by Holt \& Macdonald (1968).

† Present address: Genetic Engineering Research Department, Bristol Myers Co., P.O. Box 657, Syracuse, NY 13201, U.S.A. 


\section{Table 1. Genotypes and linkage data for the strains of Aspergillus nidulans used}

Genes determining (1) resistance : act $A$, actidione; $a c r A$, acriflavine; iod $A$, iodoacetate ; fac $A$, fluoracetate; (2) conidiospore colour: cha $A$, chartreuse; $w A$, white; $y A$, yellow; (3) nutritional requirements: $b i A$, biotin; nic $B$, nicotinamide; pabaB, p-aminobenzoic acid; phen $A$, phenylalanine; pro $A$, proline; pyro $A$, pyridoxine; $r i b o A$, riboB, riboflavin; (4) inability to utilize: $c n x A$, nitrate; gal $A$, galactose; $s C$, sulphate; (5) decreased enzyme activity: palC, alkaline phosphatase; (6) suppressor: suBpro $A$, suppresses pro $A$; (7) altered penicillin yield: pen $B$, pen $C$, raised titre; npe $B$, npe $C$, npe $D$, reduced titre; (8) morphology: sgp, slow growing non-perithecial.

\begin{tabular}{|c|c|c|c|c|c|c|c|c|}
\hline $\begin{array}{l}\text { Domestic } \\
\text { codes }\end{array}$ & $\begin{array}{l}\text { Linkage } \\
\text { group ... I I }\end{array}$ & II & III & IV & V & VI & VII & VIII \\
\hline G27 & biA & + & + & + & + & + & + & chaA \\
\hline G30 & $y A$ & acr $A$ & galA & pyro $A$ & $\operatorname{fac} A$ & $s B$ & nicB & $\mathrm{riboB}$ \\
\hline G107 & $b i A$ & $w A$ & + & penC & + & + & $n i c B$ & + \\
\hline G109 & + & $\boldsymbol{w A}$ & penB & pyro $A$ & facA & + & + & riboB \\
\hline G121 & \multicolumn{8}{|c|}{ Prototroph bearing $s g p$ (linkage group unknown) } \\
\hline GH49 & $y \boldsymbol{A}$ & + & (npeB) & pyro $A$ & + & + & + & $\operatorname{cn} x A$ \\
\hline GH50 & $y A$ & + & + & (npeC) pyroA & + & + & + & $\operatorname{cnx} A$ \\
\hline GH117 & biA & $w A(n p e D)$ & + & + & + & + & + & + \\
\hline GH119 & $b i A$ & $w A$ & + & (npeC) & + & + & + & + \\
\hline GH124 & biA & $w A$ & (npeB) & + & + & + & + & $\operatorname{cnx} A$ \\
\hline GH163 & + & $w A$ & + & $\begin{array}{l}\text { palC pabaB } \\
\text { pyroA }\end{array}$ & + & + & + & + \\
\hline GH165 & $\begin{array}{l}\text { riboA proA } \\
\text { biA }\end{array}$ & $w A$ & $\begin{array}{l}s C \text { galA } \\
\text { act } A \text { phen } A \\
\text { suBproA }\end{array}$ & + & + & + & + & + \\
\hline GH168 & + & $\operatorname{iod} A$ acr $A$ & + & + & + & + & + & + \\
\hline GH170 & $b i A$ & $w A$ & + & penc & + & + & nicB & + \\
\hline
\end{tabular}

Haploidization agents. Benlate [methyl(1-butylcarbamoyl)-2-benzimidazole carbamate] was obtained from Dupont Chemicals Co. Ltd, and Thiram (tetramethylthiuram disulphide) from BDH.

Genetic techniques. Except where indicated, parasexual and sexual crosses and the characterization of the resulting progeny were performed according to the procedures of Pontecorvo et al. (1953). The growth of segregants on diagnostic media (MM supplemented with various combinations of required nutrients or $\mathrm{CM}$ with the addition of certain normally toxic chemicals) was scored numerically on a 1 to 5 scale, by reference to the growth of the wild-type (5) and the mutant strains (1) inoculated side by side on the same agar medium. These data along with the penicillin titres of each segregant were then processed by a data-sorting computer program (written by J. F. M.), which calculated and displayed the frequencies of recombination for every pairwise combination of marker genes segregating in a cross, having first assigned each segregant a particular numerical npe or pen genotype on the basis of its penicillin titre. In addition, this program could produce an analysis of the penicillin titre distribution with respect to each marker gene, and the average titre of all segregants showing a particular genotype. Strains with characteristics that were intermediate between those associated with mutant or wild-type alleles could be excluded from analysis or be processed as having either the mutant or the wild-type phenotype in consecutive runs of the program.

Penicillin vield testing. Penicillin production was assessed using the method of Holt \& Macdonald (1968).

\section{RESULTS}

The following pairs of strains were crossed parasexually: GH124 (npeB) $\times$ G30; GH119 $($ npeC) $\times \mathrm{G} 30$; and GH117 (npeD) $\times \mathrm{G} 30$. Strain G30 is labelled on all eight linkage groups. Following haploidization of the heterozygous diploids on CM containing $1 \mu \mathrm{g}$ benlate $\mathrm{ml}^{-1}$ for the first cross, or CM containing $2.5 \mu \mathrm{g}$ thiram ml-1 (Upshall \& Johnson, 1981) for the second and third crosses, segregants were isolated and tested for penicillin yield and for the genetic markers borne. The results showed that npeB was located on chromosome III, npeC on chromosome IV, and npeD on chromosome II (Table 2). The few segregants isolated showing recombination between npeB and galA and between npeD and acr $A$ or $w A$ (numbers italicized in Table 2) were probably the result of coincident mitotic crossing over.

After a sexual cross had established that npeB was more than 50 map units from galE, adI and methH, all located on the left arm of chromosome III, an analysis of the progeny from a sexual 
Table 2. Frequency of segregation of various markers on known linkage groups with npeB, npeC or npeD

Segregants were isolated following induced haploidization of the heterozygous diploids in three parasexual crosses between strains G30 and GH124 (npeB), G30 and GH119 (npeC), and G30 and GH117 (npeD). The difference in the total numbers of segregants analysed for each pair of markers results from the exclusion of segregants which were not unequivocally scored as being of either genotype (see Methods). The figures in italic are the percentage of recombinants which were probably the result of coincident mitotic crossing over.

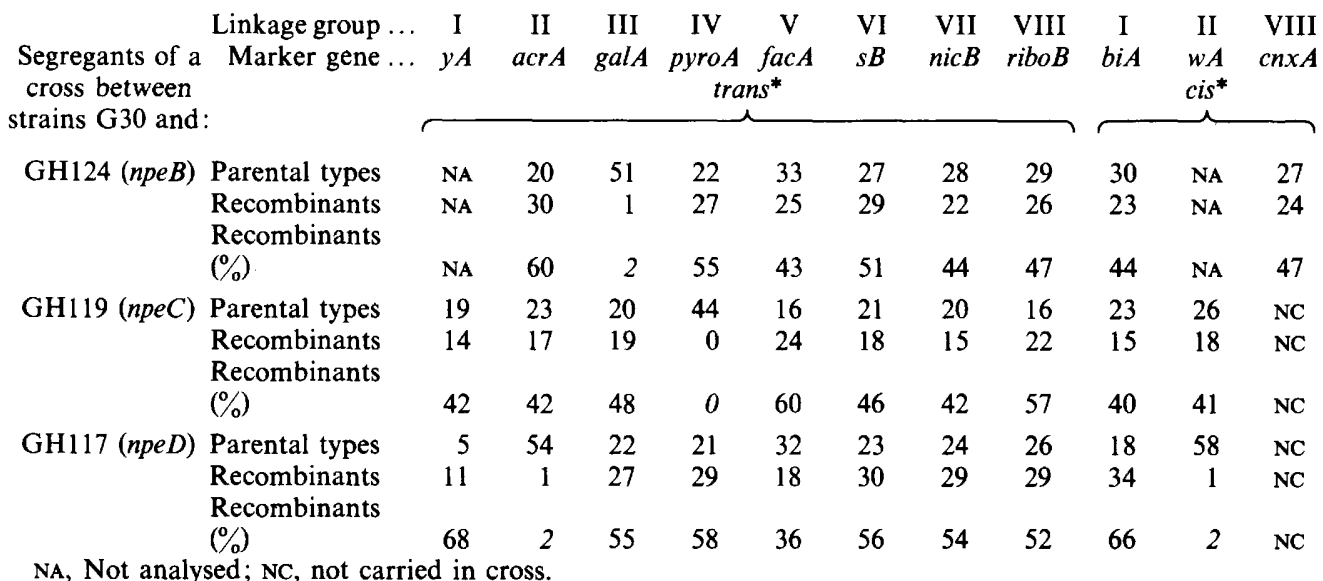

* Marker configuration with respect to the npe mutation carried in each cross.

cross between strains $\mathrm{GH} 49$ and $\mathrm{GH} 165$ indicated that $n p e B$ was closely linked to the suppressor mutation suBproA on the right arm of chromosome III. The presence of this latter marker was scored by performing test crosses between recombinants not requiring proline and strain G27 which has chartreuse-coloured conidia. Ascospores from individual cleistothecia were plated on $\mathrm{CM}$ and the resulting growth inspected for the presence of colonies with different conidial colours in ratios consistent with their having arisen from a hybrid cleistothecium. Colonies from ascospores of such hybrid cleistothecia were then tested for the presence or absence of a requirement for proline. When the proportion of proline-requiring strains to prototrophs was about one third, the original proline independence was considered to have been due to the presence of the suppressor mutations suBproA. Data from this cross provided evidence that npeB was located about 8 map units from suBproA and some 30 from phenA (see Table $3 a$ ), placing it on the same chromosome which bore pen $B$ (Ditchburn et al., 1976) but on the opposite arm.

A cross between strains GH163 and GH119 established that npeC was located between palC and pabaB on chromosome IV (Table $3 b$ ). Previous work by Ditchburn et al. (1976) had located pen $C$ on chromosome IV at a distance less than 50 units from pyro $A$. A cross between strains GH117 and GH168 located npeD at a distance of 6 units from iodA (Table $3 c$ ) confirming the suggestion of Cole (1977) that npeD was on the left arm of chromosome II.

It was decided then to set up crosses of the following types: pen $C \times n p e C$, pen $B \times n p e B$, pen $B$ $\times$ npe $C$ and pen $C \times$ npe $B$. It was envisaged that from the first cross the distribution of penicillin titres among progeny might give an estimate of linkage between penC and npeC while the other crosses would give a measure of the inherent variation in pencillin titre among progeny from crosses between strains where the mutations affecting pencillin titre were more than 50 map units apart on homologues of the same chromosome $($ pen $B \times$ npeB) or on different chromosomes (pen $B \times$ npeC and pen $C \times$ npeB).

Strains of $A$. nidulans bearing penC do not produce cleistothecia, but Ditchburn et al. (1976) showed that penC mimicked mutants designated sgp which grew slowly and did not have cleistothecia (Houghton, 1970) but where interallelic crosses yielded fertile hybrids. Therefore a sexual cross was made between strain G107 (penC nicB biA wA) and strain G121 (sgp). Since 
Table 3. Recombination frequencies among segregants from crosses made to position the mutations npeB, npeC and npeD

Values are the percentage recombination between each pair of markers carried on the same linkage group as the relevant npe mutation. The figures in italic show the percentage recovery of each marker gene among the population of segregants.

(a) Recombination frequencies of markers carried on linkage group III among 211 segregants from the sexual cross of strains GH165 and GH49 (npeB)

$\begin{array}{lcccccc} & \text { npeB } & s C & \text { galA } & \text { act } A & \text { phenA } & \text { suBpro } \\ \text { npeB } & 51 & 49 & 46 & 36 & 30 & 8 \\ \text { sC } & & 51 & 52 & 48 & 49 & 50 \\ \text { galA } & & 48 & 10 & 16 & 38 \\ \text { act } A & & & 51 & 6 & 28 \\ \text { phen } A & & & & & 41 & 22 \\ \text { suBpro } & & & & & & 53\end{array}$

(b) Recombination frequencies of markers carried on linkage group IV among 187 segregants from the sexual cross of strains GH163 and GH119 (npeC)

$\begin{array}{lcccc} & \text { npeC } & \text { palC } & \text { pabaB } & \text { pyroA } \\ \text { npeC } & 48 & 5 & 15 & 25 \\ \text { palC } & & 54 & 30 & 40 \\ \text { pabaB } & & & 46 & 10 \\ \text { pyroA } & & & & 44\end{array}$

(c) Recombination frequencies of markers carried on linkage group II among 194 segregants from the sexual cross of strains GH168 and GH117 (npeD)

$\begin{array}{lcccc} & n p e D & \text { iod } A & \text { acr } A & w A \\ \text { npeD } & 51 & 6 & 41 & 50 \\ \text { iod } A & & 51 & 35 & 50 \\ \text { acr } A & & & 48 & 25 \\ w A & & & & 50\end{array}$

Table 4. Distribution of penicillin titres among the progeny of crosses of types penC $\times$ npeC, pen $B \times$ npe $B$, pen $B \times$ npeC and pen $C \times$ npe $B$

Strains bearing $n p e B$ or $n p e C$ yielded 0.5 units penicillin $\mathrm{ml}^{-1}$, the wild-type, 6 units $\mathrm{ml}^{-1}$, strains bearing pen $B, 12$ units $\mathrm{ml}^{-1}$ and strains bearing penC, 20 units $\mathrm{ml}^{-1}$.

No. of segregants in penicillin titre groups

Cross (units $\mathrm{ml}^{-1}$ )

$\mathrm{GH} 170($ penC) $\times \mathrm{GH} 50($ npeC $)$

$\mathrm{G} 109($ penB $) \times \mathrm{GH} 49($ npeB $)$

$\mathrm{G} 109($ pen $B) \times \mathrm{GH} 50($ npeC)

$\mathrm{GH} 170($ penC) $\times \mathrm{GH} 49($ npeB $)$

\begin{tabular}{rcccc}
\multicolumn{5}{c}{$\begin{array}{l}\text { No. of segregants in penicillin titre groups } \\
\text { (units } \mathrm{ml}^{-1} \text { ) }\end{array}$} \\
\hline $0-5$ & $5-10$ & $10-15$ & $15-20$ & $>20$ \\
61 & 2 & 8 & 56 & 8 \\
75 & 8 & 56 & 60 & 0 \\
79 & 98 & 32 & 39 & 0 \\
138 & 48 & 67 & 33 & 0
\end{tabular}

neither was fertile, all the cleistothecia developing were considered to be hybrid. A pooled ascospore suspension was spread on nutritionally supplemented medium and after incubation $\left(5 \mathrm{~d}, 37^{\circ} \mathrm{C}\right)$ cleistothecia were evident on about $25 \%$ of the colonies; these were tested for penicillin yield and characterized for their auxotrophic requirements. An isolate having white conidia, requiring nicotinamide and biotin for growth, producing cleistothecia on SPM and with a penicillin titre of 16 units $\mathrm{ml}^{-1}$ was designated $\mathrm{GH} 170$. The penicillin yield of this strain indicated that it bore penC.

Sexual crosses were then made as follows: GH170 (penC) $\times$ GH50 (npeC); G109 (penB) $\times$ GH49 (npeB); G109 (penB) $\times$ GH50 (npeC) and GH170 (penC) $\times$ GH49 (npeB). The full genotypes of these strains are shown in Table 1 . Segregants were selected by their ability to grow on MM supplemented with pyridoxine and the results are given in Table 4.

While there was a broad distribution of penicillin yields among progeny from crosses of types $p e n B \times n p e C$ and pen $C \times n p e B$, only very few segregants from crosses of types pen $B \times n p e B$ and 

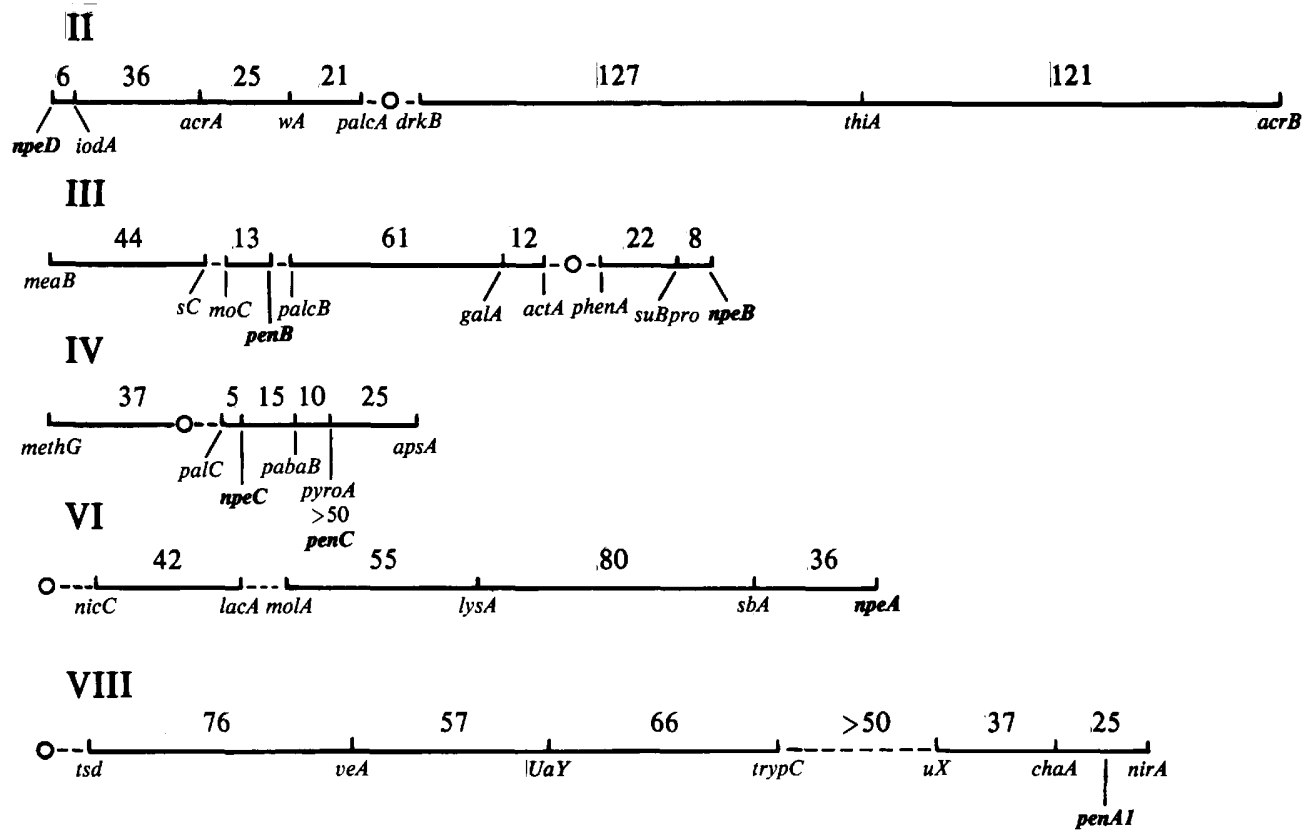

Fig. 1. Genetic map of Aspergillus nidulans. Mutations concerned with the biosynthesis and overproduction of penicillin are in bold print and a few other genetic markers are shown to allow the location of these penicillin mutations. The results from Table 3 were used for the positioning of npe and pen mutants, and those from Clutterbuck (1981) for the positioning of other genetic markers.

Explanations of symbols: $O$, centromere; npe $A, n p e B$, npe $C$, npe $D$, impairing penicillin titre; penAl, pen $B 2$, pen $C 3$, increasing penicillin titre; $a c r A, a c r B$, acriflavine-resistant; $a c t A$, actidione-resistant; aps $A$, anucleate primary sterigmata; $\operatorname{ch} a A$, chartreuse-coloured conidia; $d r k B$, dark coloured conidia; gal $A$, galactose non-utilizing; iod $A$, iodoacetate resistant; lac $A$, lactose non-utilizing; lys $A$, lysinerequiring; mea $B$, methylammonium resistant; $m e t h G$, methionine-requiring; $m o C$, morphologically abnormal; mol $A$, molybdate resistant; nic $C$, nicotinic acid-requiring; nir $A$, nitrite non-utilizing; $p a b a B$, para-aminobenzoic acid-requiring; palC, alkaline phosphatase deficient; palc $A$, palcB, acid and alkaline phosphatase deficient; phen $A$, phenylalanine-requiring; pyro $A$, pyridoxine-requiring; $s b A$, sorbitol non-utilizing; $s C$, reduced inorganic sulphur-requiring; suBpro, suppressor of proline requirement; thi $A$, thiazole-requiring; $\operatorname{tryp} C$, tryptophan-requiring; $t s D$, temperature sensitive; $u X$, urea non-utilizing; $u a Y$, uric acid non-utilizing; $v e A$, velvet morphology; $w A$, white-coloured conidia.

penC $\times$ npeC gave intermediate penicillin titres, on initial tests, to suggest that they were recombinants bearing the wild-type alleles of the pen and npe mutations. Most were low or high in penicillin yield indicting that they carried the pen or the npe mutations, respectively. Among progeny from crosses of types pen $B \times$ npe $B$ and pen $C \times n p e C$ none of these few segregants which appeared to bear the wild-type alleles of the pen and npe mutations could be classified unequivocally as having intermediate penicillin titres on repeat tests.

A heterozygous diploid was synthesized between strain GH124 bearing npeB and having a penicillin titre of $0 \cdot 1$ units $\mathrm{ml}^{-1}$ and strain $\mathrm{G} 109$ bearing pen $B$ and having a titre of $15 \mathrm{units} \mathrm{ml}^{-1}$. The diploid yielded 12 units $\mathrm{ml}^{-1}$. A second heterozygous diploid was made between strain GH50 bearing npeC, with a titre of 0.3 units $\mathrm{ml}^{-1}$ and strain $\mathrm{G} 107$ bearing penC with a titre of 16 units $\mathrm{ml}^{-1}$. It had a yield of 15 units $\mathrm{ml}^{-1}$.

\section{DISCUSSION}

Three mutations npeB, npeC and npeD, impairing penicillin production, have been located on the genetic map of $A$. nidulans. Previous investigations located mutation npeA (Holt et al., 1976), and three mutations increasing penicillin production, pen $A$, pen $B$ and pen $C$, have been mapped by Ditchburn et al. (1976). The positions of these seven mutations on the genetic map of $A$. nidulans are shown in Fig. 1. 
All four npe mutations were shown to be recessive (Edwards et al., 1974) but while penA behaved as a recessive mutation, pen $B$ was dominant and penC was semi-dominant (Ditchburn et al., 1976). Our results, where diploids were synthesized between a strain bearing npeB and one bearing pen $B$ and between a strain bearing pen $C$ and one bearing $n p e C$, were not in conflict with those of Edwards et al. (1974) or Ditchburn et al. (1976).

The inability to isolate pen ${ }^{+} n e^{+}$recombinants, as measured by their penicillin titre, from crosses between pen $B$ and npe $B$ strains is clearly not evidence of close linkage between pen $B$ and $n p e B$ as these genes have already been shown to be more than 50 map units apart. There may be processes acting against production or isolation of certain recombinants in these crosses. Consequently it is not possible to conclude that the absence of wild-type recombinants in the penC $\times$ npe $C$ cross indicates close linkage of these two genes, and the relative positioning of the two mutations awaits a more accurate mapping of penC. If the mutations penC and npeC were contiguous, it would be of considerable interest indicating that both positive and negative mutations with respect to antibiotic titre could occur at the same locus; this would offer a genetic basis for explaining earlier work with Streptomyces viridifaciens (Dulaney \& Dulaney, 1967), where it was shown that strains which reverted to production of tetracycline and were derived from mutants not yielding this antibiotic could have their antibiotic titre restored to that of the grandparent strain. What is even more important was that, in some cases, the antibiotic titres of the revertants were higher than that of the grandparent strain (Dulaney \& Dulaney, 1967; Dhar \& Bose, 1969).

That enzymes altered from their original configuration, perhaps by sequential nonsense and missense mutations, can have enhanced properties relative to the wild-type enzyme is supported by studies on the enzyme glutamate dehydrogenase in Neurospora crassa. Following the isolation of enzyme-negative mutants, revertants were selected from these, some of which yielded enzymes with increased heat stability relative to the original wild-type enzyme (Fincham, 1973). Similarly, an enzyme involved in the biosynthesis of an antibiotic could be altered by two successive mutations in such a way that the outcome was for the doubly mutated strain to elaborate more antibiotic than its grandparent strain.

This work was performed while John F. Makins was in receipt of CASE award (no. B/76/503895) from the Science Research Council in collaboration with the Procurement Executive, Ministry of Defence.

\section{REFERENCES}

Clutterbuck, A. J. (1974). Aspergillus nidulans. In Handbook of Genetics, vol. 1, pp. 447-510. Edited by R. C. King. London: Plenum Press.

Clutterbuck, A. J. (1981). Loci and linkage map of Aspergillus nidulans. Aspergillus News Letter 15, 5872.

Cole, D. S. (1977). Antibiotic production in Aspergillus nidulans. Ph.D. thesis, Polytechnic of Central London, U.K.

Cole, D. S., Holt, G. \& Macdonald, K. D. (1976). Relationship of the genetic determination of impaired penicillin production in naturally occurring strains to that in induced mutants of Aspergillus nidulans. Journal of General Microbiology 96, 423426.

DHAR, A. K. \& Bose, S. K. (1969). Mutagens for regeneration of an antibiotic producing strain of Aspergillus versicolor. Applied Microbiology 16, 340342.

Ditchburn, P., Holt, G. \& Macdonald, K. D. (1976). The genetic location of mutants increasing penicillin yield in Aspergillus nidulans. In Proceedings of the Second International Symposium on the Genetics of Industrial Micro-organisms, pp. 213-227. Edited by K. D. Macdonald. London: Academic Press.
Dulaney, E. L. \& Dulaney, D. H. (1967). Mutant populations of Streptomyces viridifaciens. Transactions of the New York Academy of Sciences 29, 782799.

Edwards, G. F. St L., Holt, G. \& Macdonald, K. D. (1974). Mutants of Aspergillus nidulans impaired in penicillin biosynthesis. Journal of General Microbiology 84, 420-422.

FinchaM, J. R. S. (1973). Genetic control of enzymeprotein structure and synthesis in fungi. In Genetics of Industrial Micro-organisms, Actinomycetes and Fungi, pp. 97-108. Edited by Z. Vanek, S. Hostalek \& J. Cudlin. Prague: Academia.

Holt, G. \& Macdonald, K. D. (1968). Penicillin production and its mode of inheritance in Aspergillus nidulans. Antonie van Leeuwenhoek 34, 409-416.

Holt, G., Edwards, G. F. St L. \& Macdonald, K. D. (1976). The genetics of mutants impaired in the biosynthesis of penicillin. In Proceedings of the Second International Symposium on the Genetics of Industrial Micro-organisms, pp. 199-211. Edited by K. D. Macdonald. London: Academic Press.

Houghton, J. A. (1970). A new class of slow growing non-perithecial mutants of Aspergillus nidulans. Genetical Research 16, 285-292. 
Macdonald, K. D. \& Holt, G. (1976). Genetics of biosynthesis and over-production of penicillin. Science Progress 63, 547-573.

Makins, J. F. \& Holt, G. (1982). Liposome-protoplast interactions. Journal of Chemical Technology and Biotechnology 32, 347-353.

Makins, J. F., Allsop, A. \& Holt, G. (1981). Intergeneric cosynthesis of penicillin by strains of Penicillium chrysogenum, $P$. chrysogenum/notatum and Aspergillus nidulans. Journal of General Microbiology 122, 339-343.

Normansell, P. J. M., Normansell, I. D. \& Holt, G.
(1980). Genetic and biochemical studies of mutants of Penicillium chrysogenum impaired in penicillin production. Journal of General Microbiology 112, 113-126.

Pontecorvo, G., Roper, J. A., Hemmons, L. H., Macdonald, K. D. \& Bufton, A. W. J. (1953). The genetics of Aspergillus nidulans. Advances in Genetics 5, 141-238.

UPShall, A. \& Johnson, P. E. (1981). Thiram induced abnormal chromosomal segregation in $A$. nidulans. Mutation Research 89, 297-301. 